\title{
Calibration sphere for low-frequency parametric sonars
}

\author{
Kenneth G. Foote ${ }^{a)}$ \\ Woods Hole Oceanographic Institution, Woods Hole, Massachusetts 02543 \\ David T. I. Francis and Philip R. Atkins \\ Department of Electronic, Electrical and Computer Engineering, University of Birmingham, Edgbaston, \\ Birmingham B15 2TT, United Kingdom
}

(Received 11 February 2006; revised 16 October 2006; accepted 17 December 2006)

\begin{abstract}
The problem of calibrating parametric sonar systems at low difference frequencies used in backscattering applications is addressed. A particular parametric sonar is considered: the Simrad TOPAS PS18 Parametric Sub-bottom Profiler. This generates difference-frequency signals in the band $0.5-6 \mathrm{kHz}$. A standard target is specified according to optimization conditions based on maximizing the target strength consistent with the target strength being independent of orientation and the target being physically manageable. The second condition is expressed as the target having an immersion weight less than $200 \mathrm{~N}$. The result is a $280-\mathrm{mm}$-diam sphere of aluminum. Its target strength varies from $-43.4 \mathrm{~dB}$ at $0.5 \mathrm{kHz}$ to $-20.2 \mathrm{~dB}$ at $6 \mathrm{kHz}$. Maximum excursions in target strength over the frequency band due to uncertainty in material properties of the sphere are of order $\pm 0.1 \mathrm{~dB}$. Maximum excursions in target strength due to variations in mass density and sound speed of the immersion medium are larger, but can be eliminated by attention to the hydrographic conditions. The results are also applicable to the standard-target calibration of conventional sonars operating at low-kilohertz frequencies. (c) 2007 Acoustical Society of America.
\end{abstract}

[DOI: 10.1121/1.2434244]

PACS number(s): 43.58.Vb, 43.30.Xm, 43.25.Zx, 43.30.Pc [AJZ] Pages: 1482-1490

\section{INTRODUCTION}

Parametric sonars are fundamentally different from conventional sonars, whose transmit signals propagate linearly, without interacting. At sufficiently high intensities, transmit signals propagating collinearly interact through the intrinsic nonlinearity of the medium, generating waves at the sum and difference frequencies. ${ }^{1,2}$ If the transmit signals consist of two waves at relatively high and similar primary frequencies, then the difference frequency will be relatively low, hence capable of propagating to relatively large ranges. Remarkably, the same difference-frequency wave possesses a directionality resembling that of the beams at the primary frequencies, but without sidelobes. In addition, modest changes in the signal frequency during transmission can generate a broad bandwidth at the difference frequency.

These properties of exceptional directionality and bandwidth at low frequencies have been exploited in a wide range of applications. One class of applications exploits the parametric sonar as a directional and/or broadband sound source in transmission over a one-way path. These include applications to shallow-water communications, ${ }^{3}$ and fish swimbladder resonance, ${ }^{4,5}$ among others. Another, larger class of applications exploits the properties of parametric sonar in backscattering, hence over a two-way path. These include acoustic scattering by the sea surface, ${ }^{6,7}$ water column, ${ }^{8}$ bottom as in the determination of geoacoustic properties ${ }^{6,9-11}$ and seafloor characterization, ${ }^{12-14}$ and seabed vegetation. ${ }^{15}$ Some other backscattering applications include sub-bottom

\footnotetext{
${ }^{a)}$ Electronic mail: kfoote@whoi.edu
}

profiling, ${ }^{16}$ detection of objects on and in the seabed, ${ }^{17-21}$ and marine archaeology. ${ }^{22,23}$ The parametric sonar has also been configured as a sidescan sonar for bottom and sub-bottom surveying. ${ }^{24}$ Rather recently it has been combined with synthetic aperture processing for detection, imaging, and classification of buried waste such as dumped ordinance in the Baltic Sea, ${ }^{25}$ and buried mines. ${ }^{26}$

Calibration is useful for most of these applications, but is seldom mentioned, much less explicitly addressed. In some of the very few cases where it is addressed, measurements have been made of the difference-frequency source level, ${ }^{4,5}$ and range and/or angular dependence of the transmit field. ${ }^{11,27}$ These measurements have been made by a hydrophone, which itself requires calibration. At other ranges and angles where knowledge of the difference-frequency field is required, models may be exercised, for example, those of Moffett and Mellon ${ }^{28}$ or by means of the Bergen Code. ${ }^{29}$

In backscattering applications, the receiver is integral to sonar performance. Given the number of quantitative backscattering applications, as well as interests in detecting and imaging objects on and beneath the seafloor, it may be wondered whether another calibration method might determine the overall response of the transmit and receive functions of the parametric sonar at low difference frequencies. In particular, might the standard-target method, which has a solid grounding in theory and in practice with high-frequency scientific echo sounders and multibeam sonars, be extended to difference-frequencies in the low-kilohertz range? If so, this would enable parametric and other low-frequency sonars to quantify scattering effects due to excitation of the powerful breathing-mode resonance of fish swimbladders ${ }^{30-34}$ in both the forward ${ }^{4,5}$ and other directions. 
Application of the standard-target method would enable the sonar to be calibrated as an integral system in which the combined transmit and receive response function is measured against a primary standard, with typical uncertainty less than $\pm 0.1 \mathrm{~dB}$ over a broad frequency range, from lowkilohertz to ultrasonic frequencies. ${ }^{35,36}$ In contrast, in hydrophone-based methods the transmit and receive parts of the sonar are measured separately, and with less accuracy because of their reference to other hydrophones or standards, whose uncertainty even at national laboratories is of order $\pm 0.5 \mathrm{~dB} .{ }^{37}$ When the overall system response function is derived from such piecewise measurements, errors also compound. Over time, the acoustic properties of robust standard targets retain their basic values; their stability over any measurement period is ensured. Hydrophones may perform stably over a period of time, but their stability over a period of a single measurement is of paramount importance. ${ }^{37,38}$ Environmental sensitivity may be an issue for both standard targets and hydrophones, but compensation is routine for standard targets; it is of more significant concern for hydrophones.

In the following, the standard-target method is briefly reviewed for high-frequency sonars in both wideband and narrowband modes. Issues peculiar to parametric sonar are identified. An optimization algorithm is then defined for specifying a robust spherical target to serve as the standard target at the difference frequencies of a particular parametric sonar, with bandwidth $0.5-6 \mathrm{kHz}$. Results of preliminary computations of target strength ${ }^{35}$ are elaborated for a single calibration target. Effects of uncertainty in knowledge of the material properties and effects due to variations in temperature and salinity of the immersion medium are described in detail. Both the magnitude and stability of target strength of the standard target are discussed.

\section{STANDARD-TARGET METHOD}

\section{A. Conventional sonar}

For conventional sonars operating linearly, the standardtarget method of calibration typically involves measurement of a chosen target at a known position in the transducer beam. ${ }^{39,40}$ This is usually on the axis and at a relatively accessible range in the transducer farfield, hence where the field quantities change inversely with range. At other ranges than that of the calibration measurement, the effect of receiver processing can be determined by a purely electronic measurement, as with a device that plays a signal of known magnitude and time delay into the transducer leads. ${ }^{41}$

One aim of such a calibration measurement is determination of the overall response $H$ of the transmit-receive system as a function of frequency $\nu$. This relates the received echo spectrum $S_{R}$ to the transmit signal spectrum $S_{T}$ by means of the backscattering form function $F$ of the standard target:

$$
S_{R}=S_{T} F H P,
$$

where $P$ expresses the dependence of the echo on the propagation path, including range and absorption if not fully com- pensated in the receiver, i.e., the two-way acoustic-path loss. ${ }^{42}$

In applications to narrowband sonar or wideband sonar in which the output is a single measure of echo strength, the constituent quantities in Eq. (1) are typically squared and integrated. The applicable operational measure of backscattering cross section of the standard target in this case is ${ }^{43}$

$$
\sigma_{1}=4 \pi \int|S F H|^{2} d \nu / \int|S H|^{2} d \nu,
$$

where the integration is performed over the entire frequency domain. When the sonar is used in a wideband mode, with the intent of determining the frequency dependence of the echo strength, the idealized single-frequency backscattering cross section of the standard target is

$$
\sigma_{2}=4 \pi|F|^{2} .
$$

This is also the limiting form of Eq. (2) when the transmit signal at $\nu_{0}$ is essentially monochromatic, with $S \propto \delta\left(\nu-\nu_{0}\right)$, or the receiver frequency response function centered at $\nu_{0}$ is ideally sharp, with $H \propto \delta\left(\nu-\nu_{0}\right)$, where $\delta$ is the Dirac delta function.

The standard-target method of calibrating sonars is widely used for narrowband scientific echo sounders operating at ultrasonic frequencies, e.g., over the range 18$710 \mathrm{kHz}^{44,45}$ It is also used for wideband scientific echo sounders, e.g., the Broadband Acoustic Scattering Signatures System, with seven octave-bandwidth transducers spanning the total frequency range $25 \mathrm{kHz}-3.2 \mathrm{MHz}{ }^{46,47}$ More recently, protocols for calibrating high-frequency multibeam sonars by the standard-target method have been worked out and reduced to practice for multibeam sonars operating at 90 , 200 , and $240 \mathrm{kHz}^{48}$

Standard targets designed and used for these systems have included precision spheres made of copper and tungsten carbide with 6\% cobalt binder. Diameters of copper spheres vary from $64 \mathrm{~mm}$ for use at $18 \mathrm{kHz}^{45}$ to $60 \mathrm{~mm}$ at $38 \mathrm{kHz}^{43}$ to $23 \mathrm{~mm}$ at $120 \mathrm{kHz}$. Diameters of tungsten carbide spheres have varied from $38.1 \mathrm{~mm}$ for use at 38,120 , and $200 \mathrm{kHz},{ }^{36,44}$ to $10 \mathrm{~mm}$ at low megahertz frequencies. ${ }^{49}$ Miyanohana et al. $^{50}$ have advised against using high-carbon steel spheres and have specified eight tungsten carbide spheres with diameters from 35 to $54.1 \mathrm{~mm}$ to span the frequency range $10-200 \mathrm{kHz}$. Corresponding target strengths of the various targets are in the approximate range from -50 to $-30 \mathrm{~dB}$.

\section{B. Parametric sonar}

The nonlinear interaction of two collinear waves that gives rise to the difference-frequency wave occurs in the immersion medium. This is significant for the particular realization of the standard-target calibration method. Nonlinear interactions also occur in the target material, but these are entirely negligible in the particular case, as argued in the Appendix.

To discuss details of the standard-target calibration method for parametric sonars, it is useful to distinguish the operating regimes of the array. As summarized by Moffett 
and Konrad, ${ }^{2}$ there are three ordinary regimes. These are differentiated by two quantities: (i) the scaled source level,

$$
\mathrm{SL}_{0, \mathrm{sc}}=\mathrm{SL}_{0}+20 \log \nu_{0}
$$

where $\mathrm{SL}_{0}$ is the source level of a primary frequency and $\nu_{0}$ is the mean primary frequency, and (ii) the absorption. This second quantity is expressed as the product of the absorption coefficient $\alpha_{0}$ at $\nu_{0}$ and the Rayleigh length

$$
R_{0}=A / \lambda,
$$

where $A$ is the area of a primary-frequency projector and $\lambda$ is the wavelength at the primary frequency. Since $R_{0}$ is a measure of the collimation length of the transmitter, $\alpha_{0} R_{0}$ measures the absorption loss in the primary-frequency nearfield.

The three regimes are the following. (i) When $\mathrm{SL}_{0, \mathrm{sc}}$ $>280 \mathrm{~dB}$ re $1 \mu \mathrm{Pa}$ at $1 \mathrm{~m}$ and $1 \mathrm{kHz}$, harmonic distortion is significant, and the difference-frequency wave is fully formed in the array nearfield, within the range $R_{0}$. (ii) When $\mathrm{SL}_{0, \mathrm{sc}}<280 \mathrm{~dB}$ re $1 \mu \mathrm{Pa}$ at $1 \mathrm{~m}$ and $1 \mathrm{kHz}$, and $\alpha_{0} R_{0}$ $>1 \mathrm{~dB}$, signal levels are relatively small and most of the absorption, hence difference-frequency generation, occurs within the range $R_{0}$. (iii) When $\mathrm{SL}_{0, \mathrm{sc}}<280 \mathrm{~dB}$ re $1 \mu \mathrm{Pa}$ at $1 \mathrm{~m}$ and $1 \mathrm{kHz}$ and $\alpha_{0} R_{0} \ll 1 \mathrm{~dB}$, most of the primaryfrequency absorption, hence difference-frequency generation, occurs in the array farfield.

In the first two cases, the array length is effectively limited. If a standard target is placed beyond the effective array length $R_{0}$, then calibration may proceed as with a conventional sonar, for the difference-frequency field is formed and varies inversely with range $r$ for $r>R_{0}$. This presumes that the particular parametric sonar is to be used for ordinary measurements outside of the interaction zone, which is a reasonable assumption for the two cases.

In the third case, the array length is generally so extended that measurements must be made within the interaction zone. In this general case, which also subsumes the first two cases when measurements are to be performed in the interaction zone, it is necessary to find a means of extrapolating to other ranges. This may be done at least partly through additional calibration measurements, but ultimately requires a computational model, for example, one established by Moffett and Mellen, ${ }^{28}$ among others, with inclusion of finite-aperture and spherical-spreading effects, ${ }^{51,52}$ as in the Bergen Code. ${ }^{29}$ Effects due to dispersion, as in the presence of air bubbles, ${ }^{53}$ may have to be included.

A key issue in the standard-target calibration of parametric sonars at low difference frequencies is finding an appropriate target. If the difference frequency is relatively high, say of order $10 \mathrm{kHz}$ or higher, then there are precedents. If the difference frequency is relatively low, say of order 1$10 \mathrm{kHz}$, then finding a suitable target presents challenges. These are addressed in the following after describing the particular parametric sonar.

\section{SPECIFICATION OF A PARAMETRIC SONAR}

For definiteness, an operational parametric sonar with low difference frequencies is chosen. This is the Simrad topographic parametric sonar TOPAS PS18 Parametric Sub- bottom Profiler. ${ }^{17}$ It is based on two primary frequencies in the range $15-21 \mathrm{kHz}$. Difference frequencies are generated over the band $0.5-6 \mathrm{kHz}$ by each of several excitations, e.g., a continuous wave or a chirp, or linear frequency sweep, of the primary signals. At $4 \mathrm{kHz}$ the secondary beam source level is at least $204 \mathrm{~dB}$ re $1 \mu \mathrm{Pa}$ at $1 \mathrm{~m}$. It can be steered over an $80^{\circ}$ sector.

The operating regime of the TOPAS parametric sonar can be inferred. The primary beamwidth is about $3.5^{\circ}$. If the array is assumed to be circular, then its radius can be determined from the formula relating the product of wave number $k$ and radius $a$, namely

$$
k a=1.615 / \sin (\Delta \theta),
$$

where $\Delta \theta$ is the half-beamwidth, namely $1.75^{\circ}$ or roughly $\pi / 103=0.0305 \mathrm{rad}$. The product $k a$ is thus about 53 , and the directivity index at $18 \mathrm{kHz}$ is $20 \log k a=34 \mathrm{~dB}$ to an excellent approximation. ${ }^{54}$ The output power $P$ is specified as being at least $32 \mathrm{~kW}$. Assuming, conservatively, that this is also the acoustic power, then

$$
\mathrm{SL}=10 \log P+\mathrm{DI}+171,
$$

or about $250 \mathrm{~dB}$ re $1 \mu \mathrm{Pa}$ at $1 \mathrm{~m}$. The scaled source level is thus $\mathrm{SL}+20 \log 18=276 \mathrm{~dB}$ re $1 \mu \mathrm{Pa}$ at $1 \mathrm{~m}$ and $1 \mathrm{kHz}$.

Secondary, difference-frequency source levels have been both calculated and measured for the mentioned TOPAS PS 18 sonar by Dybedal. ${ }^{17}$ These measurements were made for each of four frequencies, $0.5,1,2$, and $4 \mathrm{kHz}$, at each of three ranges: 40,60, and $120 \mathrm{~m}$. The calculations indicate a gradual approach to a maximum level, or saturation state, which is still not reached at $120 \mathrm{~m}$, whereas the data suggest attainment of saturation already at $60 \mathrm{~m}$ for the extreme frequencies of investigation, 0.5 and $4 \mathrm{kHz}$, but not so for the intermediate frequencies, 1 and $2 \mathrm{kHz}$.

Since $k a=53$, and $k=2 \pi / \lambda$, where $\lambda$ is the wavelength at the mean primary frequency, $18 \mathrm{kHz}$, namely $8.2 \mathrm{~cm}, a$ is about $70 \mathrm{~cm}$. The nearfield distance from Eq. (5) is $R_{0}$ $=\pi a^{2} / \lambda$, hence about $18.3 \mathrm{~m}$, which is consistent with the minimum depth of operation, namely $20 \mathrm{~m}$, as given in the manufacturer's specifications. This distance will vary with the hydrographic conditions through the sound speed, hence $\lambda$. For a temperature change from 0 to $20{ }^{\circ} \mathrm{C}$ in seawater of salinity $35 \mathrm{ppt}, \lambda$ will change from 8.05 to $8.45 \mathrm{~cm}$ and $R_{0}$ will vary from 19.1 to $18.2 \mathrm{~m}$.

The absorption coefficient at the mean primary frequency of $18 \mathrm{kHz}$ for water of temperature $10{ }^{\circ} \mathrm{C}$ and salinity $35 \mathrm{ppt}$ is about $0.0027 \mathrm{~dB} / \mathrm{m} .{ }^{55}$ Thus, the absorption at $18.3 \mathrm{~m}$, namely $\alpha_{0} R_{0}$, is about $0.05 \mathrm{~dB}$. Changes in hydrographic conditions will generally change $\alpha_{0}$, but without significantly changing the magnitude of $\alpha_{0} R_{0}$ relative to unity.

The particular parametric sonar therefore conforms to the third of the Moffett and Konrad regimes, without nearfield saturation due to harmonic distortion and with difference-frequency generation in the farfield of the primary, source arrays. As noted in Sec. II B, this corresponds to the more general case. Thus, calibration measurements with a standard target would have to be made at substantial ranges compared to those of more conventional calibrations, e.g., of high-frequency scientific echo sounders, where target 
ranges are generally less than about $25 \mathrm{~m}$. Target deployment and control at large ranges are technical challenges. Water depth, surprisingly perhaps, may be less of an issue because of the special directionality characteristics of parametric sonar, namely the narrowness of the transmit beam and absence of sidelobes. Depending on the mounting of the particular TOPAS PS18 unit, calibration could be performed in the horizontal plane. Finding a relatively distant standard target, similarly, may not present particular problems since the TOPAS PS18 beam can be steered over an $80^{\circ}$ sector. For present purposes, however, the critical problem is finding a suitable target for use over the difference-frequency range $0.5-6 \mathrm{kHz}$.

\section{TARGET OPTIMIZATION}

The problem of target optimization was originally addressed for high-frequency scientific echo sounders, ${ }^{43}$ with reference to the theory of acoustic scattering by homogeneous, solid elastic spheres ${ }^{56,57}$ or as a limiting case of scattering by homogeneous elastic shells. ${ }^{58}$ Electrolytic-grade copper was identified as being particularly suitable because of its hardness, other elastic properties, corrosion resistance, commercial availability in a high state of purity due to its worldwide use by the electrical industry, and specification of its physical properties. Variations in the backscattering form function with respect to wave number $k$ due to changes in sound speed in the immersion medium were exploited to achieve a substantially higher backscattering cross section than that of the geometric cross section. The optimization condition imposed at $38 \mathrm{kHz}$ was that the backscattering cross section be an extremum with respect to temperature and salinity over the range $[0,30]^{\circ} \mathrm{C}$ in seawater of salinity $35 \mathrm{ppt}$, while the backscattering cross section was of order $40 \mathrm{~cm}^{2}$. The result was a copper sphere of diameter $60 \mathrm{~mm}$. Subsequent optimization at this and other ultrasonic frequencies has demonstrated the suitability of tungsten carbide with $6 \%$ cobalt binder. Materials such as aluminum, brass, bronze, and steel for calibration spheres are always prima facie attractive, but knowing their exact composition and elastic properties can be problematic.

For the problem of finding a suitable target at the rather low frequencies of the TOPAS PS18 parametric sonar, several optimization conditions were considered in addition to the condition that the backscattering cross section be independent of orientation, i.e., a sphere. Briefly, the condition was imposed that the target be as acoustically powerful as possible consistent with being physically manageable. Initially, a mass limit of $10 \mathrm{~kg}$ was imposed. This condition was then relaxed in the case of aluminum to allow an immersion weight of $200 \mathrm{~N}$, or an effective mass under immersion of about $20 \mathrm{~kg}$.

Three different materials were considered, based on experience at sea with sonar targets made of each of these, although at higher, ultrasonic frequencies. These materials are aluminum alloys, copper, and tungsten carbide with $6 \%$ cobalt binder. Nominal physical properties of each, sufficient for scattering computations, are presented in Table I. The property values for aluminum alloys were derived from list-
TABLE I. Properties of three materials: mass density $\rho_{1}$, longitudinal-wave sound speed $c_{1}$, and transverse-wave sound speed $c_{2}$.

\begin{tabular}{lrll}
\hline \hline Material & $\rho_{1}\left(\mathrm{~kg} / \mathrm{m}^{3}\right)$ & $c_{1}(\mathrm{~m} / \mathrm{s})$ & $c_{2}(\mathrm{~m} / \mathrm{s})$ \\
\hline Aluminum & 2731 & 6334 & 3117 \\
Copper & 8947 & 4760 & 2288.5 \\
Tungsten carbide & 14,900 & 6853 & 4171 \\
\hline \hline
\end{tabular}

ings of mass density and elasticity moduli for each of 68 alloys, including both wrought and cast aluminum. ${ }^{59}$ The values for copper are those of the essentially pure, electrolytic grade copper of earlier experience. ${ }^{43}$ The values for tungsten carbide with $6 \%$ cobalt binder are those determined by laboratory measurement. ${ }^{60}$

The backscattering cross section was computed from the farfield backscattering form function according to Faran's theory, ${ }^{56,57}$ but with the corrections noted in Ref. 43. Since this is to be used over a very wide bandwidth but without detailed specification of receiver processing operations, the backscattering cross section is expressed according to Eq. (3). The target strength is derived from this according to

$$
\mathrm{TS}=10 \log \left[\frac{\sigma}{4 \pi r_{0}^{2}}\right],
$$

where $r_{0}$ is the reference distance of $1 \mathrm{~m}$.

\section{RESULTS AND DISCUSSION}

Initial computations for the 100-mm-diam spheres indicated quite low target strengths at the lowest frequencies in the band $0.5-6 \mathrm{kHz}$, illustrated in Table II. Larger spheres of aluminum and copper were then considered consistent with the $10 \mathrm{~kg}$ mass limit. Again, the results, shown in Table II, indicated weak target strengths at the lowest frequencies.

The mass constraint was relaxed further to allow the immersion weight of an aluminum sphere to be $200 \mathrm{~N}$. The immersed weight and effective mass of a 280-mm-diam sphere of aluminum are $192 \mathrm{~N}$ and $19.6 \mathrm{~kg}$, respectively. In this case, the nominal TS values at 0.5 and $1 \mathrm{kHz}$ are -43.4 and $-32.9 \mathrm{~dB}$, respectively. The dependence of TS on frequency is shown in Fig. 1. This assumes the nominal material property values indicated in Table I and assumed seawater properties of mass density $1027 \mathrm{~kg} / \mathrm{m}^{3}$ and sound speed $1490 \mathrm{~m} / \mathrm{s}$, corresponding to salinity $35 \mathrm{ppt}$ and temperature $10{ }^{\circ} \mathrm{C}$.

In order to assess the stability of acoustic properties of this target with respect to potential uncertainty in knowledge

TABLE II. Sphere diameter $2 a$, mass $m$, immersed effective mass $m_{\mathrm{im}}$, immersion weight $w$, and target strength values at 0.5 and $1.0 \mathrm{kHz}$.

\begin{tabular}{lcrrrrr}
\hline \hline Material & $2 a(\mathrm{~mm})$ & $m(\mathrm{~kg})$ & $m_{\mathrm{im}}(\mathrm{kg})$ & $w(\mathrm{~N})$ & $\mathrm{TS}(0.5)$ & $\mathrm{TS}(1)$ \\
\hline Aluminum & 100 & 1.430 & 0.892 & 8.8 & -69.8 & -58.0 \\
Copper & 100 & 4.685 & 4.147 & 40.7 & -67.7 & -55.8 \\
Tungsten carbide & 100 & 7.802 & 7.264 & 71.3 & -67.3 & -55.4 \\
Copper & 125 & 9.150 & 8.099 & 79.5 & -61.9 & -50.1 \\
Aluminum & 190 & 9.808 & 6.120 & 60.0 & -53.3 & -41.9 \\
Aluminum & 280 & 31.390 & 19.586 & 192.1 & -43.4 & -32.9 \\
\hline \hline
\end{tabular}




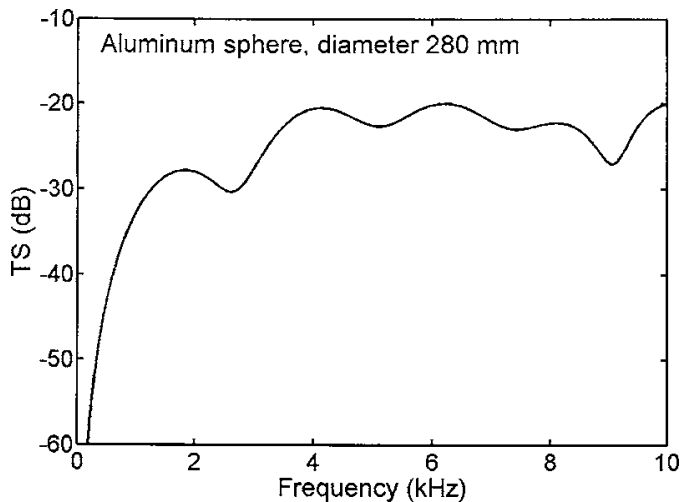

FIG. 1. Theoretical target strength spectrum of an immersed 280-mm-diam sphere of aluminum, with material properties given in Table I and assuming a medium sound speed $1490 \mathrm{~m} / \mathrm{s}$ and mass density $1027 \mathrm{~kg} / \mathrm{m}^{3}$.

of the physical properties of aluminum, $\pm 2 \%$ variations in each of the properties were considered. This is believed to be a reasonable range, based on the observed variations in mass density and elastic moduli according to the mentioned listings. ${ }^{59}$ In the case of the transverse-wave sound speed, whose influence on the acoustic scattering properties is recognized to be most sensitive, ${ }^{60-63}$ the total range of variation is $3029-3206 \mathrm{~m} / \mathrm{s}$, that is, $\pm 3 \%$, but extreme values can be excluded by avoiding the particular alloys associated with these. The nominal reference against which the comparisons were made is that of Fig. 1. Effects of uncertainty in aluminum mass density, longitudinal-wave sound speed, and transverse-wave sound speed are shown in Figs. 2-4, respectively.

Variations in target strength with respect to the aluminum mass density, Fig. 2, are seen to lie within $\pm 0.1 \mathrm{~dB}$ over nearly the entire frequency band of interest, $0.5-6 \mathrm{kHz}$, with maximum difference of about $\pm 0.12 \mathrm{~dB}$ near $2.3 \mathrm{kHz}$. Variations in target strength with respect to each of the longitudinal- and transverse-wave sound speeds, Figs. 3 and 4 , both lie within about $\pm 0.1 \mathrm{~dB}$ over the same band of interest.

Larger excursions in target strength due to potential uncertainty in the sound speeds are observed over the frequency band $6-10 \mathrm{kHz}$, with that of transverse-wave sound speed dominating that of longitudinal-wave sound speed. This is not unexpected, given earlier observations of the sen-

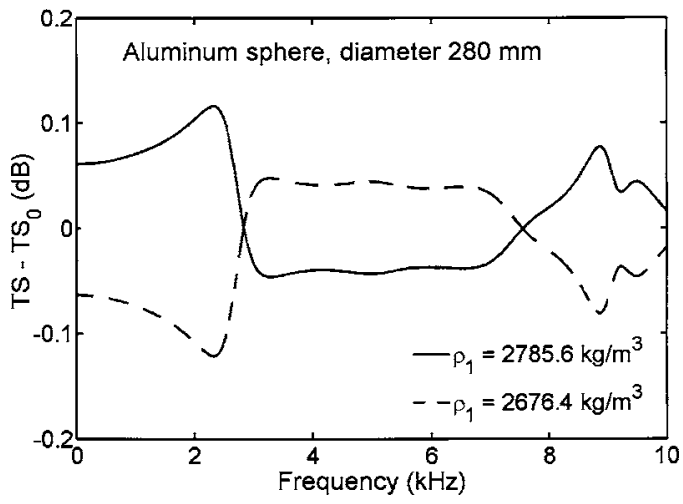

FIG. 2. Spectrum of differences in theoretical target strength of an immersed 280 -mm-diam Al sphere for $\pm 2 \%$ changes in mass density of aluminum.

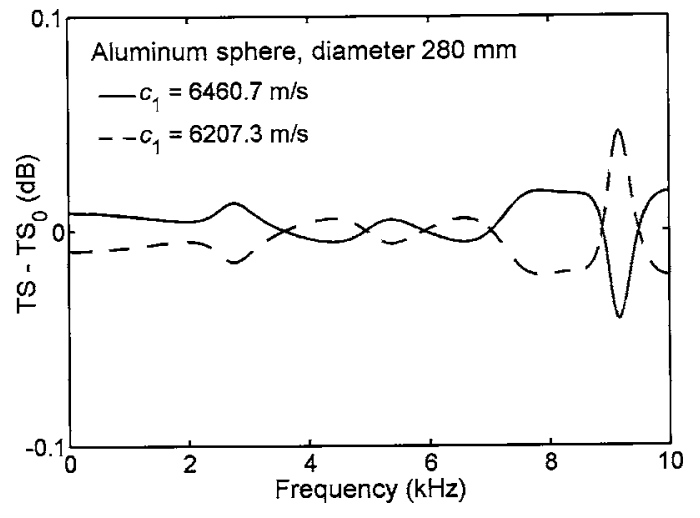

FIG. 3. Spectrum of differences in theoretical target strength of an immersed 280-mm-diam Al sphere for $\pm 2 \%$ changes in longitudinal-wave sound speed of aluminum.

sitivity of acoustic scattering to the lesser, transverse-wave sound speed in the resonance regime, as in Refs. 60-63. It might be anticipated, because free vibrations of elastic spheres, hence scattering by the same, ${ }^{56}$ are sensitive to the transverse-wave sound speed and insensitive to the longitudinal-wave sound speed. ${ }^{57,61}$

The effects of variations in properties of the immersion medium are shown in Figs. 5 and 6. In the first, the effect of variations is shown for variations in mass density over a range encompassing the cold freshwater-warm seawater range, namely $998-1032 \mathrm{~kg} / \mathrm{m}^{3}$. The greatest excursions lie within $\pm 0.2 \mathrm{~dB}$ of the nominal number.

The effect of variations in medium sound speed is shown in Fig. 6 for a $\pm 2 \%$ range of variation. The variations are as large as $\pm 0.7 \mathrm{~dB}$ over the frequency band $0.5-6 \mathrm{kHz}$; specifically, $0.69 \mathrm{~dB}$ at $3.02 \mathrm{kHz}$ and $-0.68 \mathrm{~dB}$ at $3.08 \mathrm{kHz}$. These are the largest excursions in target strength due to the variations of any property over the frequency band of interest. However, the sound speed is generally known to a very high accuracy ${ }^{64}$ during conduct of a calibration exercise, when the temperature and salinity are routinely measured, allowing compensation in the assignment of target strength. Variations in the water mass density ${ }^{65}$ are similarly known to a very high accuracy for the measured hydrography.

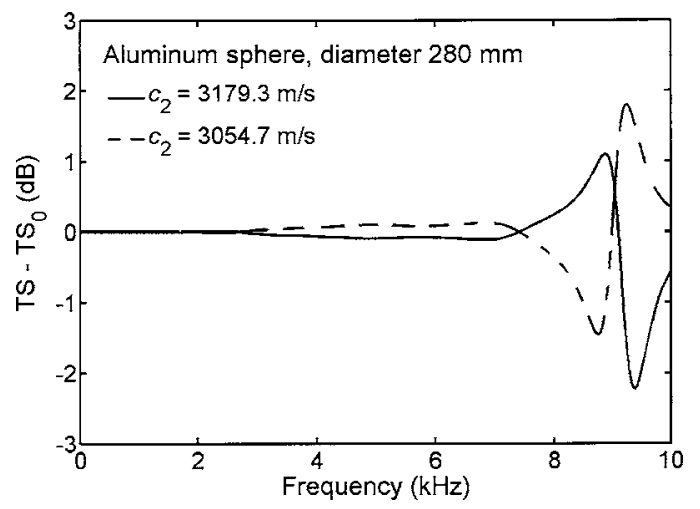

FIG. 4. Spectrum of differences in theoretical target strength of an immersed 280-mm-diam Al sphere for $\pm 2 \%$ changes in transverse-wave sound speed of aluminum. 


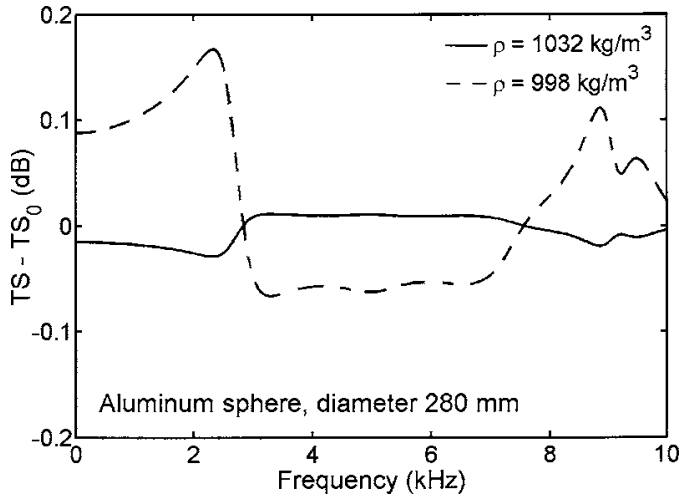

FIG. 5. Spectrum of differences in theoretical target strength of an immersed 280-mm-diam Al sphere for changes in medium mass density spanning those from cold freshwater to warm seawater, namely 998 $1032 \mathrm{~kg} / \mathrm{m} 3$.

\section{CONCLUSIONS}

The problem of calibrating parametric sonars at low difference frequencies by the standard-target method has been considered. For a particular parametric sonar, with difference frequencies in the band $0.5-6 \mathrm{kHz}$, a standard target has been specified that satisfies the conditions of having a target strength independent of target orientation and a weight in water less than $200 \mathrm{~N}$. The result is a 280 -mm-diam sphere of aluminum, with mass $31.4 \mathrm{~kg}$, immersed weight $192 \mathrm{~N}$, and effective equivalent mass in water $19.6 \mathrm{~kg}$. The total range of variation in target strength over the frequency band is $[-43.4,-20.1] \mathrm{dB}$. The greatest source of variation in target strength is that of the sound speed in the immersion medium, about $\pm 0.7 \mathrm{~dB}$ over the frequency band; however, this can be eliminated by careful attention to the hydrographic conditions. The second greatest source of variation in target strength is that of the mass density of the immersion medium, of order $\pm 0.2 \mathrm{~dB}$, which similarly can be eliminated by attention to the hydrography.

While a standard target has been specified for the performance parameters of a particular parametric sonar, the target could also be used to calibrate other sonars over a similar frequency band. Thus, conventional active sonars operating at low-kilohertz frequencies could be calibrated using the derived 280-mm-diam aluminum sphere.

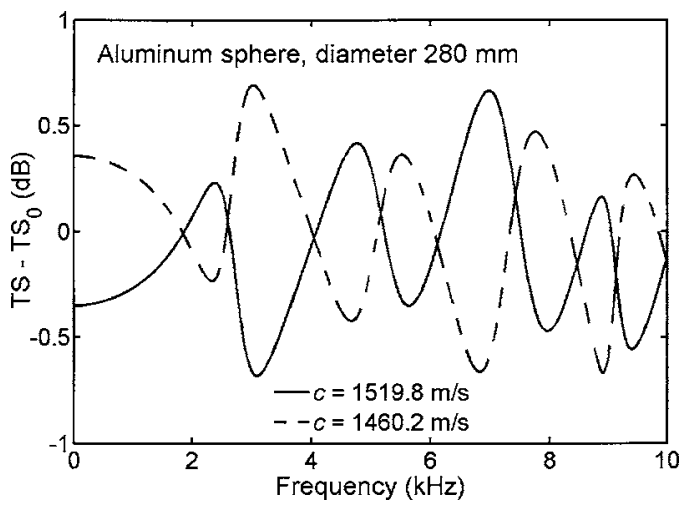

FIG. 6. Spectrum of differences in theoretical target strength of an immersed 280-mm-diam Al sphere for $\pm 2 \%$ changes in medium sound speed.
The circumstances of calibrating a particular sonar may require that the calibration be performed in the transducer nearfield when the sonar is used in the farfield or vice versa. In either case, attention must be given to the so-called nearfield problem to address extrapolation from the nearfield to the farfield and/or from the farfield to the nearfield.

\section{ACKNOWLEDGMENTS}

M. Parmenter and G. Feijoo are thanked for assistance in preparing the figures. S. Barkley is thanked for advising on electronic document compatibility.

\section{APPENDIX}

Nonlinear interactions of waves occur in solids as well as in fluids. ${ }^{66-68}$ An exact treatment of this subject would require significant effort, but this is unnecessary since it is possible to bound the effect. Initially, it is shown that secondharmonic generation is negligible for the particular target, namely the 280-mm-diam aluminum sphere, at the primary frequencies, which suggests even weaker differencefrequency generation when two harmonic waves are present.

The one-dimensional case of finite-amplitude waves in an isotropic elastic medium has been summarized succinctly by Breazeale: ${ }^{68}$

$$
\rho_{0} \frac{\partial^{2} \xi}{\partial t^{2}}=K_{2} \frac{\partial^{2} \xi}{\partial x^{2}}+\left(3 K_{2}+K_{3}\right) \frac{\partial^{2} \xi}{\partial x^{2}} \frac{\partial \xi}{\partial x},
$$

where $\rho_{0}$ is the unperturbed solid density, $\xi$ is the particle displacement, and $x$ is the distance in the direction of propagation. For an isotropic solid, the coefficients $K_{2}$ and $K_{3}$ are identical to $C_{11}$ and $C_{111}$, respectively, where the coefficients $C_{i j}$ are the ordinary second-order elastic constants, and $C_{i j k}$ are the third-order elastic constants. The second harmonic can be derived by a perturbation solution to Eq. (A1) ${ }^{69,70}$

$$
\xi=A_{1} \sin (k x-\omega t)+A_{2} \cos 2(k x-\omega t)+\cdots .
$$

Solving, $A_{2}=\beta A_{1}^{2} k^{2} x / 4$, with the nonlinearity parameter $\beta=$ $-\left(3+K_{3} / K_{2}\right) / 2$. Given the pressure amplitude $p_{1}$, the value for $A_{1}$ can be derived from the first-order wave equation, hence $A_{1}=p_{1} /\left(\rho_{0} c_{0} \omega\right)$, where $c_{0}$ is the small-amplitude longitudinal-wave sound speed, i.e., the ordinary longitudinal-wave sound speed.

For the mean primary frequency $18 \mathrm{kHz}, \omega=1.13$ $\times 10^{5} \mathrm{rad} / \mathrm{s}$. The second-order elastic constant $K_{2}=C_{11}$ is assumed to be $108 \mathrm{GPa}^{71}$ The third-order elastic constant $K_{3}$ $=C_{111}$ is derived by averaging the five values given for aluminum alloys in Ref. 72, hence $-1942 \mathrm{GPa}$. Assuming the value for mass density given in Table I, $\rho_{0}=2731 \mathrm{~kg} / \mathrm{m}^{3}$, the longitudinal-wave sound speed is $c_{0}=6289 \mathrm{~m} / \mathrm{s}$. The source level is assumed to be $250 \mathrm{~dB}$ re $1 \mu \mathrm{Pa}$ at $1 \mathrm{~m}$, as in Sec. III. If the nearest measurement to the transmitter is made at $20 \mathrm{~m}$, which is one-half the distance of the nearest measurement made by Dybedal, ${ }^{17}$ the incident pressure level is $234 \mathrm{~dB}$ re $1 \mu \mathrm{Pa}$. The corresponding pressure level is $10^{5.7} \mathrm{~Pa}=0.5 \mathrm{MPa}$ in the immersion medium and, using the series solution for the form factor, ${ }^{58}$ the displacement amplitude on the surface of the sphere facing the incident wave is 
found to be $A_{1}=2.68 \times 10^{-6} \mathrm{~m}$. The displacement amplitude decreases toward the center, so the displacement is actually greatest at the surface. If the distance of propagation in the target is taken to be the diameter, $280 \mathrm{~mm}$, then since $\beta$ $=7.5, A_{2}=1.22 \times 10^{-9} \mathrm{~m}$. That is, the displacement amplitude of the second harmonic is about $4.5 \times 10^{-4}$ that of the fundamental. This is in effect an upper limit since inspection of the solution for the displacement amplitude shows that it is greatest at the point selected.

An attempt has been made to observe the nonlinear generation of second harmonics in an available aluminum sphere of diameter $60 \mathrm{~mm}$. The sphere was suspended in the beam of a RESON broadband transducer, model TC2116, spanning the frequency band $25-150 \mathrm{kHz}$. The target range was $3.5 \mathrm{~m}$; that of the wall of the indoor tank, $7.1 \mathrm{~m}$. Amplitudeweighted sinusoidal pulses were transmitted at $100-\mathrm{Hz}$ intervals, with pulse duration $0.5 \mathrm{~ms}$, across the entire band with an insonification level of approximately $173 \mathrm{~dB}$ re $1 \mu \mathrm{Pa}$ at the target. Echoes were recorded with nominal 72-dB dynamic range. The echo signals were processed for both the fundamental, second, and third harmonics. The noise floor, as defined by the energy in the second and third harmonics, was $40-50 \mathrm{~dB}$ less than that of the fundamental echo signal due to ordinary linear backscattering by the target. Several radio signals raised this noise floor in places. Harmonic analysis of both the target and wall echoes gave similar results for the second and third harmonics. Barrett and Matsinger, ${ }^{73}$ with a similar if cleaner experimental configuration, were able to observe both sum- and differencefrequency waves due to nonlinear interaction in a crystal, but with primaries of order $0.2-1 \mathrm{GPa}$ and $7-50 \mathrm{MHz}$.

The measurements were repeated with the target sphere placed close to the projector, with an insonification level of $237 \mathrm{~dB}$ re $1 \mu \mathrm{Pa}$. Analog filtering was used to reduce the second and third harmonic levels of the drive signal such that the limit of harmonic measurement was $-60 \mathrm{~dB}$ with respect to the carrier, that is, the fundamental frequency. No detectable harmonics were introduced by the insertion of the target sphere. It is believed that the present results for the second harmonic, as well as the third harmonic, represent noise and contain no detectable signal due to the nonlinear behavior of the target sphere.

A theoretical three-dimensional approach to the nonlinear interaction of two harmonic waves in a semi-infinite solid is also available. ${ }^{74-77}$ Let $u_{1}$ and $u_{2}$ denote the displacement amplitudes of the two primary longitudinal waves incident on and just within the surface of the solid, and $k_{1}$ and $k_{2}$ denote the respective wave numbers in the solid. To a rather rough approximation, based on collinearity of all longitudinal waves in the solid, notwithstanding the angle condition required for resonance, ${ }^{74,76}$ and assumption of interaction in a spherical volume of radius $a$, the difference-frequency transverse-wave displacement amplitude is

$$
u^{(2)} \approx \frac{D u_{1} u_{2} k_{1} k_{2}\left(k_{2}-k_{1}\right) a^{2}}{3 \rho_{0} c_{0}^{2}},
$$

where $D=626 \mathrm{GPa}$ is the result of combining five elastic constants: two independent second-order constants and three independent third-order constants, required to de- scribe the elasticity of an isotropic solid, with numerical values derived from Hearmon. ${ }^{71,72}$

For an incident wave of pressure amplitude $0.5 \mathrm{MPa}$, the displacements at the primary frequencies, 15 and $21 \mathrm{kHz}$, are found from the series solution ${ }^{58}$ to be $u_{1}=2.00 \times 10^{-6} \mathrm{~m}$ and $u_{2}=1.76 \times 10^{-6} \mathrm{~m}$, respectively. Evaluation of Eq. (A3) for $a=0.14 \mathrm{~m}$ yields the estimate $u^{(2)} \approx 2.1 \times 10^{-10} \mathrm{~m}$, which compares with that of the second-harmonic displacement amplitude $A_{2}$ computed earlier. This is suggestive of a symmetry in the difference- and sum-frequency components, as with the parametric acoustic array in fluids, although inexact owing to different wave numbers at the respective frequencies. Thus the displacement amplitude of the differencefrequency wave is about $10^{-4}$ times that of the fundamental. A more meticulous treatment of the problem would undoubtedly change the estimate of $u^{(2)}$, but it is believed to within an order of magnitude. However, according to the basic theory, this result still applies to spherical waves interacting in a semi-infinite solid rather than to plane-wave-induced pressure waves interacting inside an immersed, finite, solid sphere.

${ }^{1}$ P. J. Westervelt, "Parametric acoustic array," J. Acoust. Soc. Am. 35, 535-537 (1963).

${ }^{2}$ M. B. Moffett and W. L. Konrad, Nonlinear Sources and Receivers, in Encyclopedia of Acoustics Vol. 1, edited by M. J. Crocker (Wiley, New York, 1997), pp. 607-617.

${ }^{3}$ R. F. W. Coates, M. Zheng, and L. Wang, "BASS 300 PARACOM: A model underwater parametric communication system," IEEE J. Ocean. Eng. 21, 225-232 (1996).

${ }^{4}$ O. Diachok, "Effects of absorptivity due to fish on transmission loss in shallow water," J. Acoust. Soc. Am. 105, 2107-2128 (1999).

${ }^{5}$ O. Diachok, "Absorption spectroscopy: A new approach to estimation of biomass," Fish. Res. 47, 231-244 (2000).

${ }^{6}$ W. L. Konrad, W. I. Roderick, and L. F. Carlton, "Parametric sonar as applied to high resolution boundary scattering measurement," J. Acoust. Soc. Am. 72, S41 (1982).

${ }^{7}$ B. Nuetzel, H. Herwig, J. M. Monti, and P. D. Koenigs, "A further investigation of acoustic scattering from the sea surface," J. Acoust. Soc. Am. 79, S68 (1986).

${ }^{8}$ J. M. Syck and C. L. Brown, "Volume scattering measurements using parametric sonar," J. Acoust. Soc. Am. 83, S48 (1988).

${ }^{9}$ W. I. Roderick, R. K. Dullea, and J. M. Syck, "High resolution bottom backscatter measurements," J. Acoust. Soc. Am. 75, S31 (1984).

${ }^{10}$ O. Bergem, E. Pouliquen, G. Canepa, and N. G. Pace, "Time-evolution modeling of seafloor scatter. II. Numerical and experimental evaluation," J. Acoust. Soc. Am. 105, 3142-3150 (1999).

${ }^{11}$ P. C. Hines, J. C. Osler, and D. J. MacDougald, "Acoustic backscatter measurements from littoral seabeds at shallow grazing angles at 4 and 8 kHz," J. Acoust. Soc. Am. 117, 3504-3516 (2005).

${ }^{12}$ A. Caiti, O. Bergem, E. Pouliquen, A. Biancheri, and S. Scaricaciottoli, "Seabed characterization by inversion of parametric sonar data: Selection of the cost function," in High Frequency Acoustics in Shallow Water, edited by N. G. Pace, E. Pouliquen, O. Bergem, and A. P. Lyons, SACLANTCEN Conference Proceedings Series No. CP-45 (1997), pp. 83-90.

${ }^{13}$ A. Caiti, O. Bergem, E. Pouliquen, and E. Michelozzi, "Model-based seafloor identification with parametric sonar data: Experimental results," J. Acoust. Soc. Am. 105, 1207 (1999).

${ }^{14}$ A. Caiti, J. M. Hovem, and B. Berntsen, "Quantitative characterization of the uppermost seabed stratum: Some results from the ISACS project," J. Acoust. Soc. Am. 109, 2393 (2001).

${ }^{15}$ A. P. Lyons and E. Pouliquen, "Measurements of high-frequency acoustic scattering from seabed vegetation," J. Acoust. Soc. Am. 103, 2934 (1998).

${ }^{16}$ C. C. Fox and O. L. Akervold, "Parametric acoustic arrays," J. Acoust. Soc. Am. 53, 382 (1973).

${ }^{17} \mathrm{~J}$. Dybedal, "TOPAS: Parametric end-fire array used in offshore applications," in Advances in Nonlinear Acoustics, edited by H. Hobaek (World Scientific, Singapore, 1993), pp. 264-275. 
${ }^{18}$ S. Fioravanti, A. Maguer, and A. Løvik, "A synthetic aperture parametric sonar for buried object detection," J. Acoust. Soc. Am. 100, 2636 (1996).

${ }^{19}$ W. L. J. Fox and A. Maguer, "Experimental results for detection of buried objects at low grazing angles," J. Acoust. Soc. Am. 104, 1784 (1998).

${ }^{20}$ S. Guyonic, "Buried objects detection and classification with a 3-D parametric sonar," J. Acoust. Soc. Am. 108, 2649 (2000).

${ }^{21}$ K. W. Commander, J. L. Lopes, and R. Lim, "Variation of sea-bed backscattering strength due to Bragg scattering," J. Acoust. Soc. Am. 113, 2300 (2003).

${ }^{22}$ T. G. Muir and R. S. Adair, "Potential use of parametric sonar in marine archaeology," J. Acoust. Soc. Am. 52, 122 (1972).

${ }^{23}$ W. L. Konrad and W. L. Clay, "Discovery of a sunken vessel by parametric sonar," J. Acoust. Soc. Am. 67, S67 (1980).

${ }^{24}$ W. T. Wood, J. F. Gettrust, M. K. Sen, and J. G. Kosalas, "Bottom/ subbottom surveying using a new, parametric, sidescan sonar," in High Frequency Acoustics in Shallow Water, edited by N. G. Pace, E. Pouliquen, O. Bergem, and A. P. Lyons, SACLANTCEN Conference Proceedings Series No. CP-45 (1997), pp. 607-612.

${ }^{25}$ M. E. Zakharia, S. Hibral, C. Pollet, and E. Rigaud, "Parametric synthetic interferometric sonar for bottom and subbottom imaging and mapping," J. Acoust. Soc. Am. 116, 2577 (2004)

${ }^{26}$ M. Amate, A. Hétet, S. Guyonic, M. Legris, R. Bellec, F. Maussang, J Chanussot, P. Cervenka, and J. Marchal, "Buried mines detection and classification: Advanced technologies and signal processing," Proceedings IEEE Oceans 2005 Europe, Vol. 1, pp. 153-159.

${ }^{27} \mathrm{O}$. Bergem and N. G. Pace, "Installation and calibration of a parametric array for shallow water backscatter measurements," Proceedings Oceans 1996 MTS/IEEE Conference, pp. 773-778.

${ }^{28}$ M. B. Moffett and R. H. Mellen, "Model for parametric acoustic sources," J. Acoust. Soc. Am. 61, 325-337 (1977).

${ }^{29}$ J. Berntsen, "Numerical calculations of finite amplitude sound beams," in Frontiers of Nonlinear Acoustics, Proceedings of the 12th International Symposium on Nonlinear Acoustics (Elsevier, London, 1990), pp. 191196.

${ }^{30}$ D. E. Weston, Sound Propagation in the Presence of Bladder Fish, in Underwater Acoustics Vol. 2, edited by V. M. Albers (Plenum, New York, 1967), pp. 55-88.

${ }^{31}$ B. S. McCartney and A. R. Stubbs, "Measurements of target strength of fish in dorsal aspect, including swimbladder resonance," J. Sound Vib. 15, 397-420 (1971).

${ }^{32}$ R. H. Love, "Resonant acoustic scattering by swimbladder-bearing fish," J. Acoust. Soc. Am. 64, 571-580 (1978).

${ }^{33}$ A. Løvik and J. M. Hovem, "An experimental investigation of swimbladder resonance in fishes," J. Acoust. Soc. Am. 66, 850-854 (1979).

${ }^{34}$ C. Feuillade and R. W. Nero, "A viscous-elastic swimbladder model for describing enhanced-frequency resonance scattering from fish,"J. Acoust. Soc. Am. 103, 3245-3255 (1998).

${ }^{35}$ K. G. Foote and D. T. I. Francis, "Scheme for parametric sonar calibration by standard target," in Proceedings Oceans 2005 MTS/IEEE Conference, $6 \mathrm{pp}$.

${ }^{36}$ K. G. Foote and D. N. MacLennan, "Comparison of copper and tungsten carbide calibration spheres," J. Acoust. Soc. Am. 75, 612-616 (1984).

${ }^{37}$ S. P. Robinson, P. M. Harris, J. Ablitt, G. Hayman, A. Thompson, A. L. van Buren, J. F. Zalesak, R. M. Drake, A. E. Isaev, A. M. Enyakov, C. Purcell, Z. Houqing, W. Yuebing, Z. Yue, P. Botha, and D. Krüger,"An international key comparison of free-field hydrophone calibrations in the frequency range 1 to $500 \mathrm{kHz}$," J. Acoust. Soc. Am. 120, 1366-1373 (2006).

${ }^{38}$ R. J. Bobber, Underwater electroacoustic measurements (Naval Research Laboratory, Washington, DC, 1970).

${ }^{39}$ K. G. Foote, "Maintaining precision calibrations with optimal copper spheres," J. Acoust. Soc. Am. 73, 1054-1063 (1983).

${ }^{40}$ K. G. Foote, H. P. Knudsen, G. Vestnes, D. N. MacLennan, and E. J. Simmonds, "Calibration of acoustic instruments for fish density estimation: A practical guide," ICES Cooperative Research Rep. No. 144, 1987, $69 \mathrm{pp}$.

${ }^{41}$ H. P. Knudsen, "T-A-F: Time-amplitude-frequency: A special electronic unit for measuring the TVG function in research echo-sounders," Fiskeridir. Skr., Ser. Havunders. 17, 529-541 (1985).

${ }^{42}$ K. G. Foote, "Standard-target calibration of broadband sonars," J. Acoust. Soc. Am. 108, 2484 (2000).

${ }^{43}$ K. G. Foote, "Optimizing copper spheres for precision calibration of hydroacoustic equipment," J. Acoust. Soc. Am. 71, 742-747 (1982).

${ }^{44}$ K. G. Foote, "Spheres for calibrating an eleven-frequency acoustic mea- surement system," J. Cons., Cons. Int. Explor. Mer 46, 284-286 (1990).

${ }^{45} \mathrm{~K}$. G. Foote, "Calibrating a narrowband $18-\mathrm{kHz}$ sonar," Proceedings of the MTS/IEEE Oceans 2001 Conference, pp. 2503-2505.

${ }^{46}$ K. G. Foote, T. Knutsen, P. R. Atkins, C. Bongiovanni, D. T. I. Francis, P. K. Eriksen, and T. Mortensen, "A seven-octave-bandwidth echo sounding system for application to fish and zooplankton," Acust. Acta Acust. 85, S68 (1999); J. Acoust. Soc. Am. 105, 994 (1999).

${ }^{47}$ K. G. Foote, P. R. Atkins, D. T. I. Francis, and T. Knutsen, "Measuring echo spectra of marine organisms over a wide bandwidth," in Proceedings of the International Conference on Underwater Acoustic Measurements: Technologies and Results, edited by J. S. Papadakis and L. Bjørnø, Heraklion, Crete, Greece, 28 June-1 July 2005, pp. 501-508.

${ }^{48}$ K. G. Foote, D. Chu, T. R. Hammar, K. C. Baldwin, L. A. Mayer, L. C. Hufnagle, Jr., and J. M. Jech, "Protocols for calibrating multibeam sonar," J. Acoust. Soc. Am. 117, 2013-2027 (2005).

${ }^{49}$ K. G. Foote, D. T. I. Francis, H. Furset, and H. Hobæk, "Spheres for calibrating high-frequency broadband echo sounders," J. Acoust. Soc. Am. 110, 1112-1113 (1999).

${ }^{50}$ Y. Miyanohana, K. Ishii, and M. Furusawa, "Spheres to calibrate echo sounders at any frequency," Nippon Suisan Gakkaishi 59, 933-942 (1993).

${ }^{51}$ J. N. Tjøtta and S. Tjøtta, "Effects of finite aperture in a parametric acoustic array," J. Acoust. Soc. Am. 68, 970-972 (1980).

${ }^{52} \mathrm{~J}$. N. Tjøtta and S. Tjøtta, "Nonlinear interaction of two collinear, spherically spreading sound beams," J. Acoust. Soc. Am. 67, 484-490 (1980).

${ }^{53}$ M. F. Hamilton, "Parametric acoustic array formation in dispersive fluids," J. Acoust. Soc. Am. 76, 1474-1492 (1984).

${ }^{54}$ R. J. Urick, Principles of Underwater Sound (McGraw-Hill, New York, 1983).

${ }^{55}$ R. E. Francois and G. R. Garrison, "Sound absorption based on ocean measurements. II. Boric acid contribution and equation for total absorption," J. Acoust. Soc. Am. 72, 1879-1890 (1982).

${ }^{56}$ J. J. Faran, Jr., "Sound scattering by solid cylinders and spheres," J. Acoust. Soc. Am. 23, 405-418 (1951).

${ }^{57}$ R. Hickling, "Analysis of echoes from a solid elastic sphere in water," J. Acoust. Soc. Am. 34, 1582-1592 (1962).

${ }^{58}$ R. R. Goodman and R. Stern, "Reflection and transmission of sound by elastic spherical shells," J. Acoust. Soc. Am. 34, 338-344 (1962).

${ }^{59}$ ASM Committee on Aluminum and Aluminum Alloys, Metals handbook, 9th ed. Properties and Selection: Nonferrous Alloys and Pure Metals (American Society for Metals, Metals Park, OH, 1979), Vol. 2.

${ }^{60}$ D. N. MacLennan and J. R. Dunn, "Estimation of sound velocities from resonance measurements on tungsten carbide calibration spheres," J. Sound Vib. 97, 321-331 (1984).

${ }^{61}$ W. G. Neubauer, R. H. Vogt, and L. R. Dragonette, "Acoustic reflection from elastic spheres. I. Steady-state signals," J. Acoust. Soc. Am. 55, $1123-1129$ (1974)

${ }^{62}$ K. G. Foote, "Refined determination of the rigidity modulus by echo sounder measurement," in 1981 Ultrasonics Symposium Proceedings (IEEE, New York, 1981), pp. 900-903.

${ }^{63}$ K. G. Foote, "Acoustic resonance determination of the rigidity modulus," J. Acoust. Soc. Am. 110, 2628 (2001).

${ }^{64} \mathrm{~K}$. V. Mackenzie, "Nine-term equation for sound speed in the oceans," J. Acoust. Soc. Am. 70, 807-812 (1981).

${ }^{65}$ G. Dietrich, Ozeanographie: Physikalische Eigenschaften des Meerwassers ("Oceanography: Physical Properties of Sea Water"), in LandoltBörnstein Zahlenwerte und Funktionen (Numerical Values and Functional Relationships), 6th ed., Vol. 3 (Springer, Berlin, 1952), pp. 426-440.

${ }^{66}$ R. T. Beyer, "Nonlinear Acoustics," in Physical Acoustics: Principles and Methods, edited by W. P. Mason (Academic, New York, 1965), Vol. II, pt. B.

${ }^{67}$ R. T. Beyer, Nonlinear Acoustics (Naval Ship Systems Command, Washington, DC, 1974).

${ }^{68}$ M. A. Breazeale, "Finite-amplitude waves in solids," in Encyclopedia of Acoustics, edited by M. J. Crocker (Wiley, New York, 1997), Vol. 1, pp. 227-235.

${ }^{69}$ J. Melngailis, A. A. Maradudin, and A. Seeger, "Diffraction of light by ultrasound in anharmonic crystals," Phys. Rev. 131, 1972-1975 (1963).

${ }^{70} \mathrm{M}$. A. Breazeale and J. Ford, "Ultrasonic studies of the nonlinear behavior of solids," J. Appl. Phys. 36, 3486-3490 (1965).

${ }^{71}$ R. F. S. Hearmon, "The elastic constants of crystals and other anisotropic materials," in Landolt-Börnstein Zahlenwerte und Funktionen aus Naturwissenschaften und Technik (Numerical Values and Functional Relationships in Science and Technology), New Series, Group III (Springer, Berlin, 
1979), Vol. 11, pp. 1-244.

${ }^{72}$ R. F. S. Hearmon, "The third- and higher-order elastic constants," in Landolt-Börnstein Zahlenwerte und Funktionen asu Naturwissenschaften und Technik (Numerical Values and Functional Relationships in Science and Technology), New Series, Group III (Springer, Berlin, 1979), Vol. 11, pp. 245-286.

${ }^{73}$ H. H. Barrett and J. H. Matsinger, "Interaction of almost-collinear longitudinal phonons," Phys. Rev. 154, 877-886 (1967).
${ }^{74}$ G. L. Jones and D. R. Kobett, "Interaction of elastic waves in an isotropic solid," J. Acoust. Soc. Am. 35, 5-10 (1963).

${ }^{75}$ P. Waldow and F. R. Rollins, Jr., "Remarks on a paper by Jones and Kobett," J. Acoust. Soc. Am. 35, 1060-1061 (1963).

${ }^{76}$ J. D. Childress and C. G. Hambrick, "Interactions between elastic waves in an isotropic solid," Phys. Rev. 136, A411-A418 (1964).

${ }^{77}$ I. A. Beresnev, "Interaction of two spherical elastic waves in a nonlinear five-constant medium," J. Acoust. Soc. Am. 94, 3400-3404 (1993). 